\title{
La masculinidad huichol en reacomodo para el costumbre*
}

\section{Readjusting Huichol Masculinity for el costumbre}

\author{
José de la Luz Mota-Pérez ${ }^{\text {** }}$ \\ Universidad Autónoma del Estado de México, Toluca, México. \\ Ivonne Vizcarra-Bordi*** \\ Universidad Autónoma del Estado de México, Toluca, México.
}

Recibido: 5 de febrero de 2018. Aprobado: 18 de marzo de 2018.

DOI: $10.25100 /$ lamanzanadeladiscordia.v13i1.6706

Artículo de investigación

\begin{abstract}
Resumen: Los estudios sobre masculinidades en poblaciones indígenas son exiguos y en el caso de los huicholes casi nulos, el objetivo del artículo es exponer cómo un grupo de familias de San José el Tesorero Mezquitic, Jalisco, en 2014 eligieron a dos mujeres para ocupar el cargo de "guías o cantadoras" de la peregrinación anual al desierto de Real de Catorce. Se trata de un estudio de caso que se contrapone a las etnografias clásicas y contemporáneas sobre la compleja jerarquía patriarcal de cargos rituales, civiles y católicos huicholes. Las adecuaciones en las asignaciones de género para proseguir con el costumbre de peregrinaje huichol, para este caso, puede significar una ventana que da ca-
\end{abstract}

bida a nuevas relaciones de género, y que a su vez sean propulsores del inicio de cambios sociales no sólo entre hombres y mujeres sino en los referentes a lo femenino y lo masculino constituidos en su propia cosmovisión. El estudio de caso que aqui se presenta repara en esta reflexión, retomando la religión, las instituciones y las propias relaciones de género en el peregrinaje. Es a través del reacomodo de roles, que observamos un proceso de legitimación de nuevas masculinidades huicholes.

Palabras clave: Masculinidades; Huicholes; peregrinación; migración; ritual.

Abstract: Studies about masculinities in indigenous

*Este artículo extrajo parte de la experiencia etnográfica (trabajo de campo) realizada en el marco de la Tesis Doctoral titulada "El maíz entre los huicholes: implicaciones ambientales, económicas y socioculturales en San Andrés Cohamiata, Mezquitic, Jalisco, México", pero que no conformó parte de los resultados de ésta por no corresponder a sus objetivos. La investigación se realizó gracias al financiamiento del proyecto de investigación de Ciencia Básica SEP-CONACYT No. de Referencia CB2009-130947 "El maíz mesoamericano y sus escenarios de desarrollo local".

Agradecimientos: La investigación se realizó gracias al financiamiento del proyecto de investigación de Ciencia Básica SEP-CONACYT No. de Referencia CB2009-130947 "El maíz mesoamericano y sus escenarios de desarrollo local".

**Doctor en Ciencias Agropecuarias y Recursos Naturales en la Universidad Autónoma del Estado de México, cuenta con estudios de Maestría en Ciencias Ambientales, Maestría en Estudios Mesoamericanos y Licenciatura en Antropología Social. Su línea de investigación es la etnografía de los pueblos originarios, ha realizado trabajo de campo con: otomíes del Estado de México, nahuas de Veracruz, chontales de Tabasco, mayas de Yucatán, mazahuas del Estado de México y Huicholes de Jalisco. Sus líneas de investigación incluyen la Antropología Social, las Ciencias Ambientales, los procesos rituales y la cosmovisión en Mesoamérica. Ha impartido clases en la Universidad Intercultural del Estado de México en la Licenciatura de Comunicación Intercultural. Correo electrónico: venadocolibri@yahoo.com.

***Doctora en Antropología y Maestría en Economía Rural por la Universidad Laval Quebec. Licenciada en Administración en la Universidad Autónoma Metropolitana y diversos diplomados en estudios de género. Investigadora del Instituto de Ciencias Agropecuarias y Rurales de la Universidad Autónoma del Estado de México (UAEM) desde 1986. Principales líneas de investigación: género y seguridad alimentaria. Así mismo se especializa en mejorar las metodologías cualitativas como etnografía multisitios, etnografía institucional, sistemas complejos y transdisciplinariedad. Fue vocal, vice-presidenta y presidenta de la Asociación Mexicana de Estudios Rurales. Miembro del Sistema Nacional de Investigadores Nivel III. Fue Premio Estatal de Ciencia y Tecnología en el 2010 del estado de México y Premio a la mejor investigación del campo mexicano 2001. Último proyecto de investigación "El maíz mesoamericano y sus escenarios de desarrollo local" y reciente coordinación de libro "Volteando la tortilla: Género y maíz en la alimentación actual en México". Correo electrónico: ivbordi@yahoo.com.mx. 
populations are scarce and in the case of the Huichol, there are almost none. The aim of this article is to show how a group of families from San Jose el Tesorero Mezquitic, Jalisco, elected two women to take "the position of guides or cantadoras" in the annual pilgrimage to the Real de Catorce desert in 2014. This case study stands against the classical and contemporary ethnographies on the complex patriarchal hierarchy of ritual, civil and Catholic Huichol positions. Taking participant observation as the point of departure, this ethnography emphasizes through a gender perspective the deconstruction of binary relations and their multiple combinations: women/men, nature/belief systems, women/men/other non-Huichol-indigenous and mestizos, among others, that fail to be perceived in the ethnographic horizon. The adjustments in the allocations of gender in order to continue with el costumbre of Huichol pilgrimage might represent a window that can give way to new gender relations and promote the beginning of social change not only among men and women, but also in terms of the references to the feminine and the masculine as constituted in Huichol worldviews. It is through the readjustment of roles that we bear witness to the legitimization of new Huichol masculinities.

Keywords: Masculinity; Huichol indigenous; pilgrimage; migration; ritual.

\section{a. Introducción}

Al igual que otros pueblos indígenas, el pueblo huichol ha tenido momentos en su historia para fortalecer el sistema de creencias patriarcales que contribuye a las identidades masculinas: tales como la evangelización por imposición, la formación de núcleos agrarios y ejidos, el proceso de integración nacional y en la última década ha recibido influencias del fenómeno de la globalización, de los procesos migratorios y de la educación escolarizada, entre otras injerencias institucionalizadas gubernamentales y no gubernamentales. Todo ello ha impactado directamente en las relaciones de género y, por consiguiente, en todas las formas organizativas que reproducen la vida comunitaria y hasta la esfera de lo sagrado o de la ritualidad. Específicamente es de nuestro interés responder al siguiente interrogante ¿Cómo la migración laboral ha empujado a reacomodar los rituales practicados en el peregrinaje a Real del Catorce, donde la masculinidad huichol consigue reforzarse generación tras generación? Se trata de un estudio de caso que documentamos en los años 2014, 2015 y 2016 cuando por prime- ra vez un grupo de peregrinos de San José el Tesorero, Mezquitic, Jalisco; eligieron a dos mujeres para ocupar el cargo de guías. Según el costumbre huichol, la peregrinación a Wirikuta de los grupos parentales debía ser guiada por el especialista del ritual quien hasta ese año siempre había sido un hombre (Mara 'akamel), puesto que se trata del gran rito de paso (Gutiérrez, 2002), cuyo proceso entrelaza la construcción de la identidad huichol con la sociedad, la cultura, el chamanismo y la agricultura, allí se destaca el maíz como el cultivo que rige el calendario de la vida huichol (Mota, Rivera y Vizcarra, 2017). Además, resulta interesante saber si la migración ha influenciado la toma de decisiones que ha permitido al grupo de peregrinos de tres $X i$ rikite $^{2}$, elegir a dos mujeres (doña Ángela y doña Concepción) para guiar la procesión y si ello también dio lugar a adjudicarles el cargo de cantadoras ${ }^{3}$ durante cinco años seguidos. Cabe entonces cuestionarse si este evento es un episodio de reacomodo para seguir con la tradición del ritual o si constituye el inicio de una formación de nuevas masculinidades huicholes, cuya resignificación proviene del reacomodo de roles y otras relaciones de género en el seno del grupo doméstico.

El estudio de caso se desarrolló en un grupo parental en particular. Difícilmente puede generalizarse a toda la comunidad de estudio, pues se logró constatar que la mayoría de los peregrinos huicholes no han recibido en buenos términos estos arreglos desaprobando, retando, burlándose o mostrando indiferencia ante ellos.

No obstante, creemos que a partir de este caso, se pueden generar otras pistas de investigación sobre masculinidades indígenas, entendiéndolas como la posibilidad de romper epistemes que subordinan lo indígena por su etnia y clase, y que, desde ahí, se

\footnotetext{
${ }^{1}$ Mara'akame: chamán, cantador, curandero, encargado de dirigir las ceremonias, la peregrinación.

${ }^{2}$ Los xirikite: son los oratorios familiares dedicados a los dioses de la familia, la diosa del maíz, los santos católicos y los antepasados. En la organización territorial son los lugares de vivienda o ranchos huicholes en donde residen las familias extensas, los tres xirikite analizados se integran por 48 miembros entre mujeres y hombres. La palabra $X i-$ rikite se divide en xiriki: familia nuclear o unidad doméstica y "te" es el plural, así Xirikite seria grupo de familias o unidades domésticas que habitan en un territorio común o adyacente.

${ }^{3}$ Las mujeres que conocen la compleja mitología, el sistema de cargos y saben cantar, no son llamadas mara'akmaes, de acuerdo con los informantes les falta saber realizar curaciones chamánicas y ser reconocidas por el Consejo de Ancianos.
} 
reproducen los patrones de comportamiento tradicional de masculinidad normativa, dominante y hegemónica (Díaz-Cervantes, 2014). Esta ruptura se inscribe a la necesidad de abordar y propiciar transformaciones en los hombres como estrategia hacia la equidad de género (Aguayo y Sadler, 2011; Faur, 2004; Figueroa y Franzoni, 2011), sin dejar de lado su definición como sujetos de cambio en sus prácticas masculinas; tales como paternidad, sexualidad, sociabilidad y no violencias (Connell, 2003; DíazCervantes, 2014).

De hecho, la mayoría de los estudios sobre la cultura Huichol han privilegiado el costumbrismo en diferentes esferas de la vida y cosmogonía del ser Huichol: desde las artesanías (Durin, 2008), el chamanismo (Lemaistre, 2003; Porras, 2003), la peregrinación a Wirikuta (Gutiérrez, 2002), el sacrificio de animales, los ciclos rituales (Neurath, 2002), hasta los mismos procesos de cambio en las relaciones de sociales, en las actividades económicas, así como en los aportes de las mujeres a los rituales, la economía y la feminización del trabajo artesanal (Kindl, 2003). La escasez de investigaciones que aborden la construcción identitaria de mujeres y hombres huicholes se ha convertido en un reto, porque los estereotipos sobre la cultura Huichol (Fikes, 2009; Medina, 1974) han tergiversado las relaciones de género, al interior y exterior de la comunidad.

El reto se intensifica debido a los ámbitos en los que actualmente se relacionan hombres y mujeres huicholes, tales como: el conflicto internacional sobre la apertura de minería a cielo abierto en Wirikuta (Alfie, 2015), los problemas por destitución de tierras (Liffman, 2012; Téllez, 2005), la educación prescolar, primaria, secundaria y preparatoria $(\mathrm{Du}-$ rin y Rojas, 2005), contextos de migración laboral y comercialización de artesanías y su cultura (Durin, 2008); así como en los procesos de integración o modernizadores incentivados por políticas estatales y nacionales (COPLAMAR, 1978).

\section{b. Metodología}

En el marco del estudio de la ritualidad y el maíz del pueblo Huichol, llamó la atención el constructo social de la masculinidad durante las peregrinaciones a Wirikuta en 2014, 2015 y 2016. La observación participante fue fundamental para dar cuenta de los elementos que intervienen en ese proceso, así como las entrevistas que realizamos a los hombres que participan en la peregrinación del grupo parental estudiado (48 individuos, 23 mujeres y 25 hombres), con edades que van desde los 15 hasta los 70 años, donde las mujeres y los hombres se dedican a la agricultura, a la comercialización de artesanías y a cumplir con el costumbre, por lo que emigran temporalmente. La observación y las entrevistas se realizaron en San José el Tesorero, municipio de Mezquitic, Jalisco; siendo el espacio predilecto para ello los hogares de las personas participantes. Además de hacer presencia en todas las festividades y rituales, el equipo de investigación también les acompañó a las faenas del campo para observar la división sexual del trabajo y a lugares de comercialización de artesanías para conocer sus actividades complementarias.

Como ya se mencionó, la investigación inició en el ámbito ritual-festivo comunitario, las visitas frecuentes durante 2014 y 2015 permitieron observar en el ámbito público y privado la construcción y reconstrucción social de las identidades masculinas del grupo de peregrinos estudiado.

Para tratar de explicar la masculinidad huichol se aplicó el método cualitativo-interpretativo, analizando los actos, las relaciones con la naturaleza, lo religioso, lo social, la economía y la estructura organizativa del peregrinaje a Wirikuta. Cabe mencionar además el acompañamiento realizado a los hombres del hogar a diferentes ciudades de la República mexicana, tales como Querétaro, Guanajuato, Cuernavaca, Monterrey, Guadalajara y Ciudad de México; donde se comercializan artesanías de chaquira, lo que permitió un acercamiento significativo con los informantes. Así mismo, con la ayuda de herramientas tecnológicas se obtuvieron documentos audiovisuales y fotográficos para complementar el análisis interpretativo, entendido como discursos visuales, orales y experienciales.

Ahora bien, con la incursión de dos mujeres cantadoras como guías de un grupo de xirikite de la peregrinación a Wirikuta en 2014, y cuya responsabilidad culminará en 2018, el acompañamiento al grupo familiar se realizó en tres peregrinaciones (2014, 2015 y 2016), con lo cual se observaron las diferencias entre grupos familiares que reproducen 
el orden patriarcal, aparentemente sin cambios, pues otros grupos de peregrinos son guiados por hombres. No se niega que presenciar la experiencia y los discursos entre unos y otros, puede llevar a interpretaciones intersubjetivas que favorecen el cambio de las masculinidades huicholes. Dada esta situación, se tratará la perspectiva de género como herramienta que privilegia las relaciones de poder entre hombres y mujeres condicionadas al menos por pertenecer a una y encontrase en una edad social (Díaz-Cervantes, 2014). Dicho de esta manera y de acuerdo con la temporalidad en que se encuentra el cumplimiento del cargo de guías de peregrinación, los resultados no pueden generalizarse, pero sí aportan datos sobre el cambio en la masculinidad del grupo analizado y su versión emic a partir de la experiencia. Cabe señalar que ese cambio, requiere a priori y necesariamente que las condiciones de vida de las mujeres del grupo mejoren o estén en proceso de hacerlo, para aceptar las responsabilidades que se les asignan ajenas a su género. Por consecuente, las nuevas masculinidades no pueden ni surgir ni reconstituirse sin que las mujeres elegidas para representar esos cargos masculinos presenten rasgos de empoderamiento y estén dispuestas a adquirir nuevas experiencias que también las vayan transformando como sujetos (Sánchez-Espíndola, Chávez-Mejía y Vizcarra-Bordi, 2010). Si bien el estudio no repara en dar a conocer la experiencia de las cantadoras, reconoce que, sin estos procesos propios de la feminización, el cambio de la masculinidad que se observa no se habría presentado.

Por lo tanto, el enfoque de género es privilegiado en la exploración de las dimensiones sociales y culturales que influyen en la masculinidad huichol observada en este estudio de caso. Desde este compromiso, se describen el proceso ritual y su dinámica en el peregrinaje para dar lugar al reconocimiento de las distintas subjetividades de género, considerando las relaciones binarias y de diferenciación sexual que se construyen bajo el rigor de la cosmovisión ${ }^{4}$ huichola según es el costumbre.

\footnotetext{
${ }^{4}$ López-Austin define a la cosmovisión como: "Un hecho histórico de producción de procesos mentales inmerso en decursos de larga duración cuyo resultado es un conjunto sistémico de coherencia relativa, constituido por una red colectiva de actos mentales, con la que una entidad social, en un momento histórico dado, pretende aprehender el universo de forma holística" (López-Austin, 2016, p. 22).
}

\section{c. Antecedentes}

El costumbre, como lo llaman los y las huicholes, se refiere a la forma ritualizada en que humanos, naturaleza y lo sagrado se vinculan (Boege, 1988), para darle sentido a ser ancestral y estar en el presente. El costumbre es en sí, la cosmovisión que guía cómo se relacionan estos tres ámbitos, buscando siempre los términos de la cohesión social, individual, material y espiritual. De aquí se negocian las alianzas y los intercambios con otros hombres, otras mujeres, con la naturaleza y con lo sagrado, pero también se asignan las identidades de género implicados en: la familia, el grupo de familia, la agricultura, la peregrinación, la elaboración de ofrendas, la cacería de venado, las vestimentas, las ornamentas, las artesanías, los ciclos reproductivos y festivos. En fin, el costumbre dirige la conducta ritual que permea todas las relaciones al interior y exterior de los Xukurikate .

A diferencia del pensamiento occidental descrito por Bourdieu , Hernández y Montesinos (1998) en el cual el principio de división primordial que divide a los seres humanos en hombres y mujeres, asigna a los hombres las únicas tareas dignas de ser desempeñadas, las labores que el mundo social constituye como serias, esta ilusión original, principio indiscutido que hace al hombre verdaderamente hombre, viril, para el caso los pueblos de tradición mesoamericana, el concepto de género está ligado al de cosmovisión en donde las dualidades son fluidas y no jerarquizadas, sin embargo, los huicholes, así como otros pueblos originarios, han recibido influencias externas desde la época prehispánica y hasta nuestros días, lo que dificulta circunscribirlos a categorías de género desde un episteme occidental cartesiano, aunque determinen una suscripción andropocéntrica en las políticas públicas nacionales e internacionales (Díaz-Cervantes, 2014).

La masculinidad según Figueroa y Franzoni (2011) se define como “...un conjunto de normas, atributos, valores, funciones, significados y códigos de conducta que se suponen esenciales al hombre en una cultura determinada que dictan los patrones de socialización..." (p. 68). Aunque en los estudios

${ }^{5}$ Xukurikate o kawiteros: se traduce como jicareros, se aplica para nombrar la organización por cargos que práctica el costumbre; personas encargadas de la Peregrinación a Wirikuta; guardianes de las jícaras en los diferentes centros ceremoniales. 
de género se acierta que la masculinidad no es un asunto fijo, porque también contiene elementos femeninos (Lamas, 2015). La identidad como sentido de pertenencia y reconocimiento, además de la "construcción de la idea del ser mujer y ser hombre en una cultura" (Fuller, 2001, p. 158), por lo que debemos considerar que en las culturas indígenas, el cuerpo ${ }^{6}$ y la vestimenta son contenedores de la identidad (González, 2008; López-Austin, 1996; Pitarch, 1996), y transmisores de los códigos sobre ser femenino y/o masculino durante el ciclo de vida en contexto social y ritual, porque se complejiza la construcción social de la identidad de género en esas culturas. A la etnia también intersecta la clase, donde lo campesino-masculino cobra importancia como categoría de género (Ayala-Carrillo, 2007).

Así, por ejemplo, para los pueblos de ascendencia mesoamericana el universo social y cultural es dual, lo que da lugar a la formación de relaciones binarias, cuyos ejes constitutivos se basan en opuestos complementarios en todos los procesos dinámicos de los ciclos de la vida: naturales, sociales y cósmicos. A partir de ello, lo femenino con su naturaleza "oscura, fría, fértil, húmeda, el agua, la muerte" (López-Austin, 2015, p. 28) complementa lo masculino. De aquí que lo masculino en la cultura huichol, no se refiere sólo a los atributos físicos sexuales, sino que condensa otras cualidades como la razón, el poder, la fuerza, "lo de arriba, lo solar, lo seco, el fuego, la vida” (López-Austin, 2016, p. 77).

Siguiendo a Turner (2007), en la peregrinación y el ciclo festivo tanto mujeres como hombres realizan representaciones simbólicas que por medio de prácticas acéticas como no comer, no dormir, no tener sexo, no beber agua, logran la separación de la comunidad para reactualizar los valores, símbolos, mitos e identidad comunitarios en la fase liminar o de tránsito y culminan con la integración a la comunidad, al final el individuo y el grupo se ha transformado, estableciendo la communitas. Después de la peregrinación y de cada proceso ritual los/las participantes experimentan la communitas, una sensación de igualdad social, solidaridad y convivencia en la que cada posición social tiene algo de sagrado.

\footnotetext{
${ }^{6} \mathrm{El}$ cuerpo es una forma simbólica poderosa, una superficie en la
} cual se inscriben las reglas, jerarquías y aún más, los compromisos metafísicos de una cultura. que se refuerzan a través del lenguaje corporal (Douglas, 1982).
Con la intromisión de otros referentes de la masculinidad (por lo general occidentales y mestizos), se puede decir que la construcción de la identidad huichol y su significación binaria, viven reacomodos para reproducirse en las contradicciones entre nuevas realidades y la tradición asociada a los rituales, mitos y símbolos, tanto en la familia como a nivel comunitario. Al proponer a dos mujeres cantadoras como guías de un grupo de xirikite de la peregrinación a Wirikuta, se esbozan otras relaciones de género, que, dependiendo de los resultados, pueden llegar a la "legitimación de un nuevo patrón de masculinidad diferente al tradicional" (Valdés, 2000, p. 29).

De esta manera, los estudios de género en comunidades indígenas presentan retos epistemológicos, teóricos y prácticos que requieren de un tratamiento sugerido desde la decolonialidad. Marcos (2012) insiste para este tipo de reflexiones, en cuestionar "el isomorfismo implícito en la traslación de categorías del universo teórico moderno a las conceptualizaciones de lo femenino y lo masculino en el pensamiento mesoamericano" (p. 237), así que los posiciona de nuevo en la alteridad y en la otredad.

\section{d. Resultados y discusión}

\section{Sistema de cargos}

El territorio huichol está constituido por los significados ancestrales que establecen alianzas entre la naturaleza, el pueblo y lo sagrado. Si bien, se pueden identificar geopolíticamente dónde habita el pueblo huichol (estados de Durango, Nayarit, Zacatecas, San Luis Potosí y Jalisco), sus alianzas e intercambios se realizan en torno a cinco centros ceremoniales, que no sólo mantienen el sistema de creencias, sino que dan lugar a la reproducción de complejas estructuras organizacionales de dominio patriarcal, patrilocal y parilineal (político, económico, social, familiar y territorial). En estos centros se instalan sus gobiernos tradicionales basados en Consejos de Ancianos, conforme a el costumbre, quienes finalmente definen en respeto a su cosmovisión, el cómo, cuándo y porqué de cada actividad humana. La figura 1, muestra la ubicación de los centros: $1^{\circ}$. San Andrés Cohamiata Tateikie; $2^{\circ}$. Santa Catarina Cuexcomatitián Tuapurie; $3^{\circ}$. San 
Sebastián Teponahuaxtlán Wautia; $4^{\circ}$. Tuxpan de Bolaños Tutsipa y; en Nayarit, se ubica el $5^{\circ}$, en la localidad de Guadalupe Ocotán Xatsitsarie. San Andrés Cohamiata es la localidad más importante y rige a las comunidades de: Cohamiata (Kuamiata),
San José el Tesorero (Hayukarita), Las Guayabas (Temurikita), Santa Bárbara (Kiurita), Las Pitahayas (Maaramanawe), El Chalate (Waxieti), San Miguel Huaixtita (Tsikuaita), La Laguna (Haarakuna), el Carrizal (Hakarietsie).

Fuente: Téllez (2005).

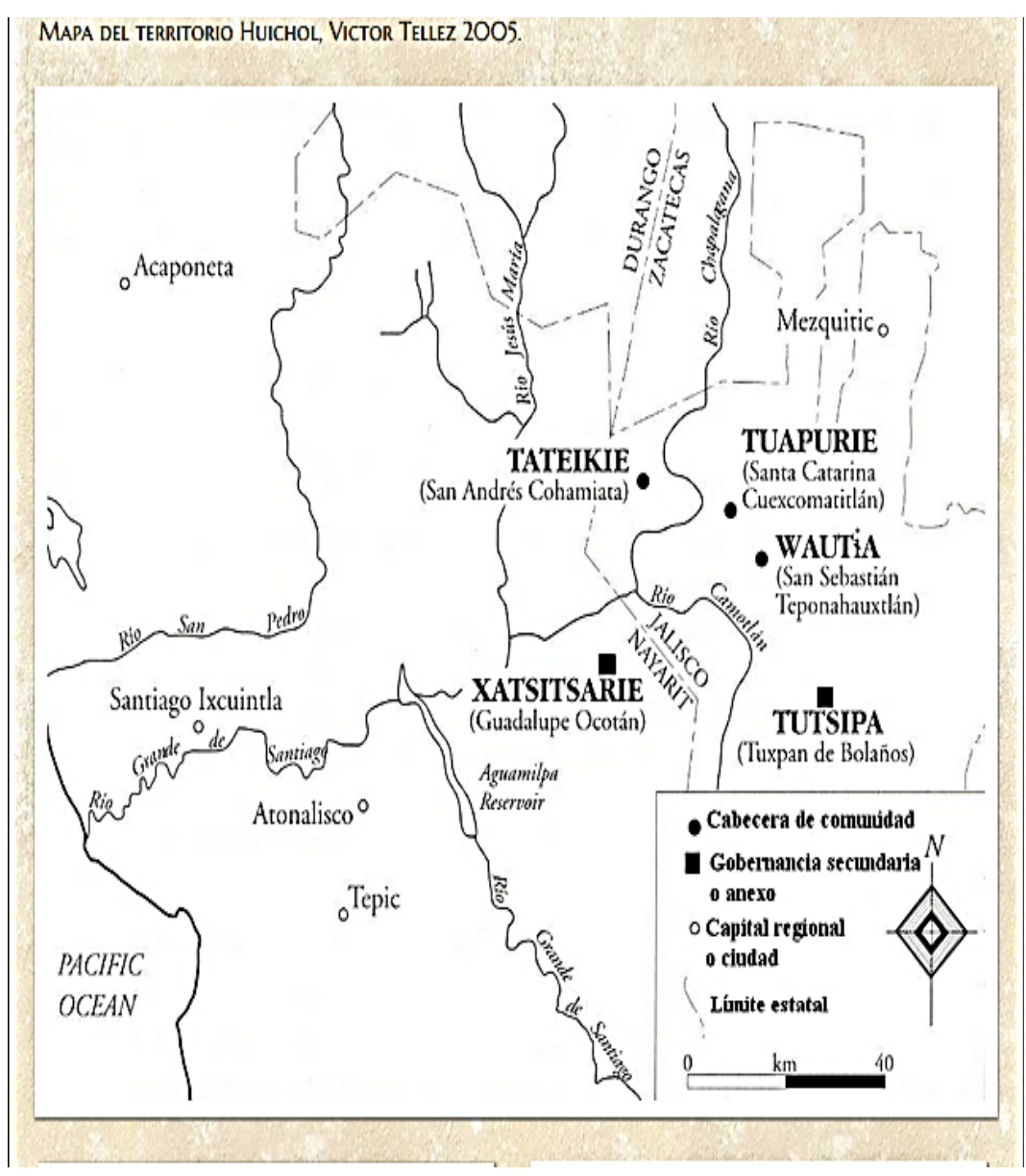

Figura 1. Las comunidades huicholas.

Los centros toman vida por las comunidades, que a su vez se encuentran articuladas en actividades colectivas identificadas como rituales o ceremonias. Ahí, los hombres "son los más fuertes, los que saben cantar, los que cumplen el costumbre, cumplen su palabra" (Ángela, comunicación personal, 17 de marzo del 2015). En los rituales, la masculinidad simboliza la vida social y la cuestión del poder comunal a través de distintas prácticas culturales como: el cultivo del maíz, la cacería de venado, la peregrinación a Wirikuta, llevar ofrendas a diferentes lugares y el ciclo festivo tradicional. Son lla- mados Xukurikate (kawiteros o jicareros), porque se encargan de cumplir con el costumbre. Corresponden a la esfera masculina, donde se toman decisiones que afectan la vida de toda la comunidad pues es la base de la organización social y territorial, la cual inicia en el Xiriki; seguido del Tukipa ${ }^{7}$ de San José, ambos subordinados a San Andrés Cohamiata que funciona como centro rector del poder $^{8}$. Aun-

\footnotetext{
${ }^{7}$ Centro ceremonial que aglutina a un conjunto de xirikite.

${ }^{8}$ Gutiérrez (2002) ha denominado circuito y distrito a las relaciones de intercambio entre los niveles de jeraquías de las comunidades huicholes. En este estudio de caso, el Circuito Tukipa comprende las nueve comunidades subordinadas a San Andrés.
} 
que no todos los hombres huichol se involucran en estas prácticas (por lo menos los pertenecientes a San José El Tesorero), las estructuras organizacionales en torno a las ceremonias se aseguran que su ausencia física sea representada simbólicamente por diferentes objetos ritualizados, como una manera de garantizar su masculinidad.

Por su parte, los mara'akames, los peregrinos, los xukurikate y el Consejo de Ancianos, son estructuras sociales y rituales que ostenta el poder, que no sólo actúan controlando la dinámica de los flujos que la constituyen, sino que también permite entender el poder cómo una derivación del control sobre los procesos y su manejo permite mayor concentración de poder.

Este régimen es observado con los xukurikate de San José el Tesorero implicados en hacer cumplir el costumbre, quienes reciben nombramientos bajo el sistema de cargos. En el caso de estudio, el sistema está conformado por un grupo organizado en 37 cargos, integrado por igual número de matrimonios, donde cada pareja cuida una jícara que representa a un dios o antepasado (Kindl, 2003). Los xukurikate se encuentran delimitados por una línea de descendencia bilateral. Es decir, por un lado, las mujeres heredan jícaras (Kindl, 2003) y los hombres reciben plumas, flechas y muwieri ${ }^{9}$ (Gutiérrez, 2002; Manzanares, 2009). Por otro lado, el Consejo de Ancianos se encarga de ubicar a las personas que recibirán los cargos por medio de linaje que pruebe que han cumplido con el costumbre. A pesar de que los cargos corresponden a la esfera masculina, las esposas tienen la gran responsabilidad de acatar el costumbre. Sin excepción, asienten que se encuentran bajo la vigilancia de un Consejo de Ancianos integrado por mara 'akate acompañados de sus esposas que no cuenta con voz ni voto en las reuniones.

Una las actividades que requiere mayor nivel de organización económica, política y ritual es la peregrinación a Wirikuta la cual se realiza anualmente durante la temporada de estiaje o seca del año ${ }^{10}$. Gutiérrez (2002) y Neurath (2002) revelan la asociación

${ }^{9}$ Muwieri: vara ceremonial con plumas de águila.

${ }^{10}$ Los primeros registros de los rituales asociados y la organización familiar fueron descritos por: Lumholtz (1981 y 1986), Preuss (Jauregui y Neurath, 1998) y Zingg (1998), pero no realizaron la peregrinación. Benítez (1968) fue el primero en acompañar a los huicholes, sin embargo, el registro más completo de la peregrinación a Wirikuta fue realizado por Gutiérrez (2002). de la peregrinación con el ciclo agrícola, la cacería de ciervos, el sacrificio de ganado vacuno, los mitos y los rituales (Figura 2). Todos estos eventos están asociados y son interdependientes y aunque se ven diferenciadas las actividades entre hombres y mujeres, individuales-familiares y comunitarias para cada época (lluvias y $\operatorname{secas}^{11}$ ), los hombres jerarquizan el sistema de organización y ostentan los cargos más importantes, sobre todo en la peregrinación. La complejidad de articular el ciclo ceremonial, el agrícola y el grupo parental en la comprensión del mundo dual, puede percibirse en el sistema de cargos de orden patriarcal. Éste domina la organización social huichol desde el núcleo familiar hasta las esferas de manifestaciones sagradas. Bajo este sistema, cada ceremonia, rito, peregrinación y actividad agrícola, artesanal, comercial y reproducción de los roles de género en la cotidianidad, estructuran el grupo parental conformado por la familia.

Fuente: Mota et al. (2017).

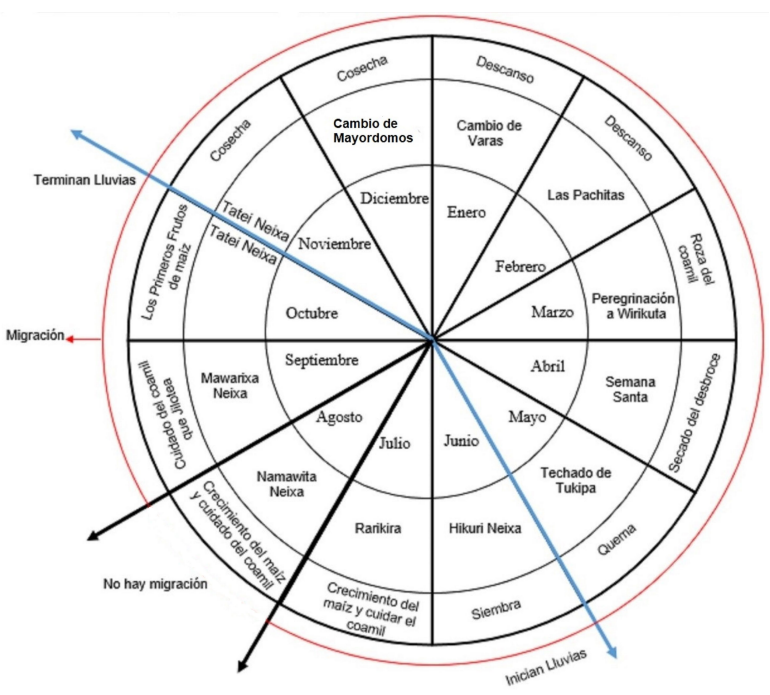

Figura 2. Articulación de los ciclos agrícola, ritual y migración.

\section{La disyuntiva}

En contextos de múltiples relaciones sociales de los distintos hombres del grupo parental con el entorno y el exterior del territorio huichol (comercialización de artesanías, acceso a mejores niveles de escolaridad formal, migración interna e interna-

\footnotetext{
${ }^{11}$ Se aclara la utilización del término secas por parte de los campesinos mexicanos para denominar el periodo del año sin lluvias.
} 
cional, trabajo remunerado extra-agrícola, etc.), los modos de vida del grupo van adquiriendo y adaptando nuevos hábitos de consumo a el costumbre. En consecuencia, la peregrinación a Wirikuta, al igual que la mayoría de las ceremonias asociadas a la ritualidad huichol, no se escapa de la adopción de elementos nuevos y de la sustitución o eliminación de otros para adecuarse a los tiempos modernos. Generalmente, los elementos modernos provienen de las transformaciones que se dan precisamente en los modos de vida de las comunidades, tales como bebidas alcohólicas, cigarrillos, bebidas gaseosas azucaradas, vestimentas, zapatos, aparatos electrodomésticos y de comunicación, así como de entretenimiento. Se trata de adaptaciones que permiten reproducir simbólicamente: los mitos, el arte y los rituales huicholes que, si bien se transforman en el tiempo y en el espacio, han logrado sostener el costumbre (Diguet, 1992; Fabila, 1956; Villa-Rojas, 1961; Weigand, 1992; Zingg, 1998).

Ante la eminente apertura, el grupo de xirikite analizados en este estudio (Tabla 1), confrontó un dilema entre permitir ser moderno y estar en la permanencia para custodiar el costumbre.

Tabla 1. Integrantes de los xirikite

\begin{tabular}{lccc}
\hline Xiriki & Mujeres & Hombres & Matrimonios \\
\hline 1. Cruz & 11 & 13 & 5 \\
2. Carrillo & 3 & 3 & 2 \\
3. López & 9 & 9 & 5 \\
Total & 23 & 25 & 12 \\
\hline
\end{tabular}

Fuente: Elaboración propia.

Debido al desfase generacional, por primera vez en este grupo, los hombres de la segunda generación y quienes ostentan el poder (Figura 3), no encontraron disposición masculina para nombrar un mara'akame en la tercera generación por lo que decidieron proponer a doña Ángela (65 años) y a doña Concepción (60 años) como guías de la peregrinación del grupo parental. El desfase generacional no se explica por diferencias de edades, sino por la disposición de la generación asignada a continuar con el costumbre. Los mara'akames quienes tienen entre los 60 y 70 años, no lograron obtener buena respuesta de la tercera generación compuesta por seis aprendices, puesto que aún se encontraban en formación inicial. Los dos casi mara'akames (de 35 y 40 años) realizaron estudios de educación superior y se han insertado con éxito al mercado laboral institucionalizado, uno en la ciudad de México y otro en Celaya, lo que les ha impedido culminar su aprendizaje para ser reconocidos por el Consejo de Ancianos de San Andrés Cohamiata. Ello no pone en evidencia la falta de capacidades de los candidatos a mara'akame, ni se cuestiona a doña Ángela y a doña Concepción en el aprendizaje de la tradición oral, los rituales, los cantos, sino que, para apegarse a la continuidad en el cumplimiento de el costumbre, resultó ser una estrategia astuta para la situación, aunque poco común. De hecho, para que los aprendices ocuparan puestos comunitarios como parte de su formación, el Consejo de Ancianos nombró a doña Ángela y a doña Concepción como guías de las peregrinaciones durante cinco años consecutivos. Tiempo suficiente para encontrar el relevo generacional, lo que vino a solucionar el problema del grupo. 
Fuente: Elaboración propia.
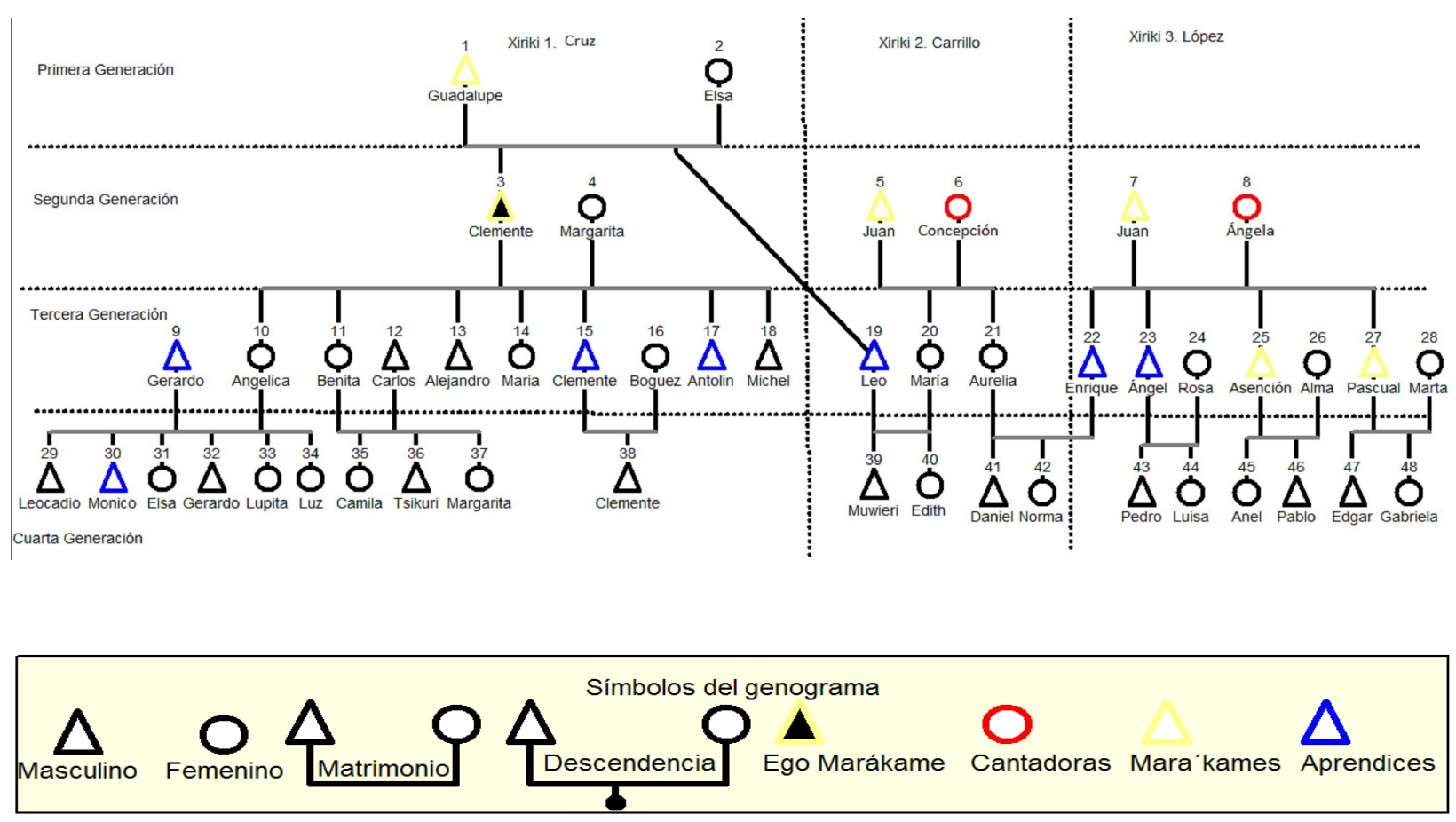

Figura 3. Integrantes y relaciones entre los xirikite.

Si bien no existía disponibilidad de los integrantes elegibles en la tercera generación, la asignación de cargos a las dos mujeres propuestas por los mara'akames también debió pasar por un proceso de apego a el costumbre, el cual consiste en que todos los cargos se conducen por un sistema onírico en el que algunos o todos los mara'akames sueñan a los que deberán ocupan los cargos, luego en sesión comunitaria el Consejo de Ancianos acepta o rechaza la propuesta. De esta manera, doña Ángela y doña Concepción pasaron por el escrutinio y fueron aceptadas al comunicarles los resultados. Son resoluciones definitivas y las asignadas no tienen capacidad de negarse, hacerlo implicaría salir del grupo y nunca volver participar en el sistema de cargos. Por otro lado, aceptarlo las dotó de mayor estatus en el grupo y no sólo con mayores responsabilidades, también tuvieron la oportunidad de mostrar sus capacidades organizativas aprendidas a través del acompañamiento de sus esposos maraka'mes de la segunda generación y junto con ello, sus conocimientos y el saber hacer la conducción de las peregrinaciones.

En efecto, para ellas no fue difícil aceptar los cargos, aunque si se mostraron preocupadas por hacer bien su trabajo. Durante cuarenta años, doña Ángela y doña Concepción han acompañado a sus esposos (Juan L. y Juan C.) en el cumplimiento de sus cargos y en cada una de las peregrinaciones. Esta experiencia junto con la transmisión oral de diferentes saberes, mitos y ritos, se convierten en las herramientas más seguras para el cumplimiento de estos cargos masculinizados.

Ahora bien, esta situación exige reacomodos en distintas dimensiones de la vida social de los tres xirikite. De los 48 integrantes, 40 se dedican a la agricultura y a la elaboración de artesanías. En la Figura 3 se muestran la organización de los xirikite, sus vínculos de parentesco, donde se observa la posición jerárquica de doña Ángela y doña Concepción (en rojo). Cuatro hombres son mara'akames (en amarillo) y dos están por obtener el rango (25 y 27 en amarrillo) y siete aprendices en azul. En el caso de la migración que se realiza en los intercisos de la inactividad agrícola y ritual, salen los seis mara'akames, dos aprendices (9 y 15) y cinco mujeres acompañantes (entre ellas Ángela y Concepción).

Entre las nuevas dinámicas sobresalen los pro- 
cesos de reconocimiento de las mujeres marginadas en espacios masculinizados. Los recientes nombramientos de doña Ángela y doña Concepción han puesto en predicación de tolerancia y aceptación a cada miembro del grupo tanto hombres como mujeres, experimentando nuevas relaciones que transforman las subjetividades de género, sobre todo las de los aprendices y los mara'akames de quienes depende la cohesión grupal. Sin duda, esta experiencia ha dado lugar a un aprendizaje de nuevas socializaciones al mirar a las mujeres desde otro nivel de jerarquía, al menos en las ceremonias y peregrinaciones.

\section{La masculinidad en el reacomodo}

La peregrinación es un rito comunitario, conformado de ofrendas prometidas a Haramara ${ }^{12}$. Éstas son llevadas por los representantes de los Xirikite, en este caso doña Ángela y doña Concepción, quienes hacen el recorrido depositándolas en la costa de Nayarit, El Cerro Gordo en Durango y, si es necesario, a Wirikuta en San Luis Potosí, Lago de Chapala en Jalisco y varias cuevas en la Sierra Madre Occidental. Deben cubrir el circuito a partir de la festividad de Tatei Neixa en noviembre y hasta junio antes de Hikuri Neixa que corresponde al inicio de las lluvias, pues la organización de la sociedad huichol gira en dos grandes periodos del ciclo agrícola el de lluvias y de sequía (Fresán, 2002; Gutiérrez, 2002; Neurath, 2002 y Manzanares, 2009).

En las tres peregrinaciones $(2014,2015$ y 2016) apoyadas por los mara'akames (3, 5, 7, 25 y 27 en la Figura 3), doña Ángela y doña Concepción dirigieron los rituales de los xirikite a través de sus cantos y acciones rituales. Para conducir la ceremonia nocturna, los mara 'akames les recordaban algún pasaje mítico y repetían sus cantos a manera de segunda voz. El apoyo también tenía el propósito de mantenerlas despiertas durante todas las ceremonias nocturnas, en 2014 fueron seis días de velaciones, en 2015 cuatro noches y en 2016 sólo tres debido al mal tiempo.

Una vez aceptadas las nuevas responsabilidades, las mujeres cantadoras (doña Ángela y doña Concepción), asentaron que, al momento de recibir el cargo de guías de la peregrinación, se comprome-

\footnotetext{
${ }^{12}$ Haramara: el mar en la costa de San Blas en Nayarit.
}

terían, al igual que lo han hecho siempre los hombres, a acudir durante cinco años a todas las celebraciones que marca el calendario de San José, el cual varía año con año, dependiendo del ciclo de las lluvias, ya que las celebraciones están sujetas al ciclo agrícola del maíz ${ }^{13}$. Para el año de 2014 el calendario fue: Corte de Tarima y postes de Tuki y Cambio de Varas en enero; entre febrero y abril se realizaron La Peregrinación, Las Pachitas y la Semana Santa; en mayo fue la Renovación de Tukipa; en junio se dieron las celebraciones de Hikuri Neixa, Namawita Neixa y Rarikira, acompañadas del Volteado de Mesa; de septiembre a octubre se presenció Mawarixa Neixa; en noviembre se vuelve al Volteado de Mesa junto con Tatei Neixa y la fiesta Patronal de San Andrés; en diciembre al final de la cosecha de maíz se realiza el Cambio de Mayordomos. Cada celebración tiene su propia ritualidad, donde la representación dual marca el mundo de lo masculino y de lo femenino.

En la peregrinación se observó que los/las participantes pueden recolectar agua de los manantiales y hikuri, pero no pueden usarlos ritualmente. Habitualmente, quienes desempeñaban los cargos de guía eran hombres, no existían tensiones de género en legitimar el uso de estos elementos para practicar los rituales de el costumbre. Sin embargo, con las nuevas asignaciones de estos cargos a doña Ángela y doña Concepción, los peregrinos han tenido que lidiar con conflictos de representación que nunca antes habían vivido. El proceso de aceptación no ha sido fácil, pero estas mujeres se han desempeñado conforme a lo acostumbrado, facilitando de cierta manera una aceptación temporal, porque en los imaginarios de los grupos que participan, existe la certeza que ello sólo es por un tiempo determinado, mientras los aspirantes culminan sus aprendizajes y entrenamientos. Pese a esa perspectiva, las mujeres han marcado un cambio significativo en la resignificación de la masculinidad, al menos en la peregrinación a Wirikuta.

De esta manera, el copal, pinole, agua, cer-

${ }^{13}$ Neurath (2002) documenta que el agua de los manantiales del desierto representa la diosa de serpiente de lluvia de oriente Nia'ariwame y el peyote (hikuri) es considerado el maíz de los antepasados, por ello la gran fiesta de Hikuli Neixa, "la danza del Peyote", es celebrada al final de la temporada de sequía y marca el inicio del ciclo agrícola del maíz. 
veza, sangre de res y venado, mazorcas de maíz, jícaras votivas, cuadros de estambre, artesanías de chaquira, espejos, cruz con monedas pegadas, autos de juguete, instrumentos musicales en miniatura y velas con las que participaron en cada ceremonia nocturna, resultan ser vehículos de recodificación en las relaciones de género. De hecho, este proceso ya existe en el seno del ritual, pues los miembros de la familia (todos y todas) que no pueden acudir a la peregrinación, son representados por los granos de maíz incluyendo a los antepasados, donde se desdibuja el género.

Otro gesto que vislumbra un pequeño cambio se percibe en el grupo de peregrinos integrado por varios xirikite y un jefe de peregrinación escogido por el Consejo de Ancianos, es decir por el mara'akame principal, quien realiza cantos en todas las ceremonias nocturnas, que deben ser repetidos por todos los mara'akate representantes de xirikite, incluidas doña Ángela y doña Concepción. Dentro de el costumbre, se requiere de una energía masculina para repetir el canto por casi toda la noche, sin importar el cuerpo que lo sostiene, de ahí asienten que las mujeres que ahora participan como cantadoras son sólo transmisoras del poder masculino. Aún con ello, después de la presentación grupal, cada grupo parental se separa a velar su propio fuego en un espacio adyacente hasta antes del amanecer, momento en que se reúnen en el fuego principal para cerrar cada ceremonia. Por parte del grupo de xirikite guiado por doña Ángela y doña Concepción, además de seguir el guion, acomodaron y presentaron las ofrendas de los peregrinos de los xirikite Cruz, Carrillo y López, incluyendo los encargos de los miembros ausentes, así como sus peticiones.

Cabe señalar que doña Ángela y doña Concepción deben asistir a otra serie de celebraciones movibles como la renovación o techado del Tukipa que es cada cinco años, aunque no es necesario que acudan a la fiesta patronal de San Andrés si deben estar presentes en todas las celebraciones del Tuki y de Cabecera. Su presencia es necesaria para mostrar las ofrendas del grupo de xirikite que representan. El número de hombres del grupo que acuden a la peregrinación varía entre 10 y 20 , reconocen en ellas la capacidad de cantar, de aguantar el desvelo, la sed, el hambre, la abstinencia de sal, abstinencia sexual y conocer la mitología, es decir conocen el costumbre huichol.

El rito de paso termina hasta que se realice la cacería de uno o varios venados, en 2014 se reorganizaron grupos de diez personas en cada caso acompañados de un mara'akame cantador, del grupo parental analizado acudieron doña Ángela, Doña Concepción y cinco hombres (3, 5, 7, 25 y 27 observados en la Figura 3).

Por otro lado, cuando las mujeres cantadoras acudieron a la cacería de venado para velar, cuidar y cantar frente al fuego de los xirikite, se dieron a la tarea de cantar durante el tiempo que duraron las actividades cinegéticas, con cantos que ubicaban el lugar de la presa y, además, se cree que con sus cantos atrajeron las presas hacia los cazadores.

Ahora bien, tanto para doña Ángela como para doña Concepción, el costumbre no representa un problema, pues cuentan con el apoyo del resto de hombres del grupo parental para cumplir con lo asignado. La preocupación mayor viene de la incapacidad para cubrir los gastos de participar en todo el ciclo festivo, agrícola e inclusive en la cacería. Estos se estiman cuantiosos, alrededor de cien mil pesos anuales. Una manifestación más de la masculinidad huichol es esa capacidad de moverse para obtener los recursos monetarios suficientes para asegurar la realización de cada celebración. La manera en que los obtienen es a partir de la comercialización de artesanías de chaquira ${ }^{14}$ y los rituales de sanación (chamánica y curandería). Con el dinero en efectivo pagan las ofrendas, compran velas, animales para sacrificio y otros artículos.

El reacomodo para solventar los gastos se dio gracias a la comprensión de los mara'akate que conforman los tres Xiriku del grupo parental (Figura 3). Ellos negociaron con la familia extensa para que auxiliaran a doña Ángela y doña Concepción a realizar todo el ciclo ceremonial, incluyendo un soporte económico extra para ellas.

Al considerar a las familias que integran el grupo de xirikite como unidades de producción y consumo (Chaianov, 1974), cuando no hay eventos rituales, las mujeres preparan los alimentos, lavan la ropa,

14 Los hombres se mueven con facilidad para vender las artesanías entre Guadalajara, Ciudad de México, Estado de México, Guanajuato, Michoacán, Morelos y Querétaro. 
cuidan a los niños, bordan ropa y morrales. En tanto que los hombres cortan la leña, desgranan el maíz, elaboran artesanías de chaquira, preparan la tierra para la siembra y emigran para comercializar sus artesanías. Se trata de una división sexual del trabajo, donde los xirikite son considerados como campesinos-artesanos (Dinerman, 1974; Papousek, 1982; Sandoval, 1994) con un fuerte componente patriarcal que se extiende hasta el orden simbólico. Así entonces, la elaboración de objetos rituales como jícaras es exclusiva de las mujeres y en el caso de las flechas y muwieri sólo son fabricadas-por hombres.

En el plano espiritual, uno de los rasgos que más caracteriza a quien se asume huichol (estructurado en grupo de xirikite) es el chamanismo ${ }^{15}$, cuya práctica es exclusiva de la esfera masculina (mara'akate). Ellos están habilitados a realizar curas o limpias, y a cambio reciben dinero en efectivo que destinan en su mayoría a cubrir los gastos del ciclo festivo y en menor medida las necesidades de la familia y la comunidad cuando atienden síndromes de filiación cultural. Pese al predominio masculino en la representación espiritual, cuando se trata de artesanías de chaquira, morrales, ropa, jícaras y flechas destinadas a la comercialización, los límites de la división sexual no son claros debido a la falta de significados en los rituales.

A diferencia de la división sexual del trabajo en el hogar, donde las mujeres se encargan de la reproducción y los hombres de la producción, en las ceremonias de peregrinación ambos tienen las mismas tareas para asegurar la ritualidad en el costumbre. Por ejemplo, a Ángela y Concepción junto con otras mujeres (4 y 20) y tres hombres $(19,22$ y 23), se les encomendó recolectar leña y mantener encendido el fuego ceremonial. Así mismo, tanto mujeres como hombres participan en la limpieza de los lugares.

Al encontrarse en una situación liminal los hombres prepararon los alimentos que se distribuyeron entre los/las asistentes. Si bien las mujeres (4, 14, $20,24,26$ y 28) extienden algunas de las actividades que se consideran propias de la esfera femenina al ámbito de la ritualidad, como acarrear agua y preparar la comida que sirve como ofrenda, doña Án-

\footnotetext{
${ }^{15}$ La literatura sobre el chamanismo huichol es abundante véase: Benítez (1973), Fikes (2009), Fresan (2002), Furst (1980), Gutiérrez (2002), Jáuregui (1998), Kindl (2003), Lemaistre (2003), Lumholtz (1981; 1986), Neurath (2002), Porras (2003), Zingg (1998).
}

gela y doña Concepción al final de cada ceremonia tienen que dar un mensaje de los dioses (el cual depende de la petición u ofrenda presentada) al grupo a través de la palabra y los cantos en huichol.

El trabajo de cantar durante las ceremonias nocturnas es agotador; inicia a las 10:00 p.m. y termina a las 7:00 a.m. por lo que las cantadoras necesitan el apoyo de otros hombres y hasta otros mara'akames para lograr el cometido. Este gesto es novedoso entre las prácticas masculinas, pues no existen registros de mujeres dirigiendo este ritual. Con esta predisposición, los hombres procuran constantemente en auxiliar a Ángela y Concepción para llegar al cierre de la ceremonia. Son detalles que ellas conocían, pero del lado del público. Ahora ellas eran las protagonistas, realizaron cantos y usaron por primera vez el muwieri (vara ceremonial con plumas) para pasar las plumas sobre las personas y las cosas presentes en el acto. Una vez que se apagó el fuego ceremonial y quedó limpio el lugar, ellas pudieron dormir sobre el suelo tapadas con una cobija. Cabe señalar, que mientras que las cantadoras asumen y aprenden su nueva asignación, los hombres que dejaron de ser guías en este grupo parental no han adquirido roles del ámbito femenino, principalmente en lo doméstico o reproductivo, pero esto no quiere decir que de alguna manera no estén considerando revalorar ese trabajo femenino, tal y como lo hizo saber el hijo de Concepción: "Yo no podría con tanta carga" (Enrique L., comunicación personal, 15 de junio de 2016), y es que Ángela, Concepción y muchas mujeres mayores de 50 años, que ya no están en edad reproductiva ni criando a sus hijos e hijas, además de realizar las tareas propias de su género como el bordado de manta, cocinar, lavar ropa, etc., realizan los mismos trabajos que los hombres, tales como elaborar artesanías de chaquira, ayudar en la siembra de maíz y migran para comercializar sus artesanías y las de otras mujeres del grupo parental que mantienen su relación bajo el dominio patriarcal.

\section{e. Conclusiones}

El objetivo del artículo fue exponer cómo se reacomoda la masculinidad en un grupo de familias huicholas de San José el Tesorero que mantienen vínculos parentales, rituales y territoriales (xirikite). 
Si bien esta es una condición que anuncia cambios importantes en las relaciones de género del pueblo huichol, elegir a mujeres en puestos exclusivamente masculinos para asegurar la continuidad de el costumbre, sólo se ha registrado como un estudio de caso al interior de un xiriki.

La vida cotidiana huichol tradicionalmente está mediada por las relaciones de género en una dinámica que se construye en la interacción social entre grupos de xirikite y dentro de ellos, que a su vez regulan las asignaciones bajo una cosmovisión dual. Sin embargo, los contextos de acceso a la escolaridad formal, a la necesidad de tener más ingresos para reproducir sus ceremonias y a confrontar otras condiciones que afecta sus modos de vida, van reacomodando las relaciones sociales y de género y, por lo tanto, reconstruyendo lentamente las identidades marcadas por el binarismo. La experiencia individual de hombres y mujeres que se refleja en la formación del grupo de peregrinos que eligió a dos mujeres (doña Concepción y doña Ángela) como guías constituyen una pista que se asoma en esos cambios, que, a nivel comunitario, inician un proceso de apertura (experimentación) que forma parte de los procesos políticos, religiosos y sociales comunitarios. De resultar favorable, se convierte en un elemento de cambio clave para incidir en los intereses de otros grupos de peregrinos y en caso contrario, el desprestigio social.

Los problemas económicos, sociales, la educación escolarizada, la migración laboral, junto con otras intromisiones que llegan a sus vidas y que aquí no se abordaron, pero que deben abordarse en futuros estudios (tales los programas sociales gubernamentales) han influido sin lugar a dudas, en las relaciones de género que se gestan en el seno de la familia extendida. En este estudio de caso observamos nuevas identidades y subjetividades de género, que impactarán en las generaciones subsecuentes y en los ámbitos domésticos, público y en los procesos rituales o espirituales.

Finalmente, para comprender los reacomodos huicholes que se dan en el ámbito de la ritualidad dominada por hombres, deben reconocerse que no sólo han surgido para resolver problemas emergentes a falta de mara'akate formados para el relevo generacional, sino que también la experiencia de in- cluir mujeres en las tareas masculinizads y jerarquizadas quedará en la memoria del grupo estudiado (xirikite), lo que será un nuevo referente para legitimar pequeños cambios en las relaciones de género sin atentar a su referente del todo organizador: su cosmovisión en el costumbre, donde la lengua, la vestimenta, la religión, los rituales, los aspectos socioeconómicos, la cultura y el proceso histórico seguirán teniendo un sentido de ser y permanecer.

\section{Referencias bibliográficas}

Aguayo, Francisco y Sadler, Michelle (Eds.). (2011). Masculinidades y Políticas Públicas. Involucrando hombres en la equidad de género. Santiago de Chile: Universidad de Chile.

Alfie, Miriam. (2015). Conflictos socio-ambientales: la minería en Wirikuta y Cananea. El Cotidiano, Sociedad y Medio Ambiente, (191), 197-108.

Ayala-Carrillo, María. (2007). Masculinidades en el campo. Ra Ximhai, (3), 739-761. Recuperado de http:// www.redalyc.org/articulo.oa?id=46130306.

Benítez, Fernando. (1968). Los indios de México. Tomo II. Los Huicholes. México: Ediciones Era.

Benítez, Fernando. (1973). Historia de un Chaman Cora. México: Ediciones Era.

Boege, Eckart. (1988). Los mazatecos ante la nación. Contradicciones de la identidad étnica en el México actual. México: Siglo XXI Editores.

Bourdieu, Pierre; Hernández, Alonso y Montesinos, Rafael. (1998). La masculinidad. Aspectos sociales y culturales. Quito, Ecuador: Abya-Yala.

COPLAMAR. (1978). Programa Integrado 9. Región HUICOT de Jalisco. México: Presidencia de la República.

Connell, Robert. (2003). Masculinidades (Irene Artigas, Trad.). México: Universidad Nacional Autónoma de México. (Obra original publicada en 1993).

Chaianov, Alexander. (1974). La organización de la unidad económica campesina. Buenos Aires, Argentina: Nueva Visión.

Díaz-Cervantes, Rufino. (2014). La perspectiva de género en la comprensión de la masculinidad y la sobrevivencia indígena en México. Agricultura, Sociedad y Desarrollo, 11(3), 359-378.

Diguet, León. (1992). Por tierras occidentales entre sierras y barrancas. México: Centro de Estudios Mexicanos y Centroamericanos.

Dinerman, Ina. (1974). Los tarascos: Campesinos y artesanos de Michoacán. México: Sep-Setentas.

Douglas, Mary. (1982). Natural Symbols. New York: Oantheon.

Durin, Severine y Rojas, Angélica. (2005). El conflicto entre la escuela y la cultura huichola. Traslape y ne- 
gociación de tiempos. Relaciones. Estudios de Historia y Sociedad, XXVI(101), 148-190.

Durin, Severine. (2008). El arte de vender artesanías. El caso de los artesanos huicholes en Monterrey. En Entre luces y sombras. Miradas sobre los indigenas en el área metropolitana de Monterrey (pp. 335-386). México: Ciesas-CDI.

Fabila, Alonso. (1956). Los Huicholes de Jalisco. México: INI.

Faur, Eleonor. (2004). Masculinidades y desarrollo social. Las relaciones de género desde la perspectiva de los hombres. Bogotá: Arango Editores y UNICEF.

Figueroa, Juan y Franzoni, Josefina. (2011). Del hombre proveedor al hombre emocional: construyendo nuevos significados de la masculinidad entre hombres mexicanos. En Francisco Aguayo y Michelle Sadler (Eds.), Masculinidades y políticas públicas: involucrando hombres en la equidad de género (pp. 64-81). Santiago de Chile: Facultad de Ciencias Sociales, Universidad de Chile.

Fikes, Jay. (2009). Carlos Castañeda. Oportunismo académico y los psicodélicos años sesenta. USA: Xlibris.

Fresán, Mariana. (2002). Nierika: una ventana al mundo de los antepasados. México: INAH.

Fuller, Norma. (2001). Masculinidad cambios y permanencias. Lima: Pontificia Universidad Católica de Perú.

Furst, Peter. (1980). Alucinógenos y cultura. México: FCE.

González, Blanca. (2008). Una lectura del cuerpo humano como entidad biológica y simbólica en el Gran Nayar. México: INAH, IIA y UNAM.

Gutiérrez, Arturo. (2002). La Peregrinación a Wirikuta: el gran rito de paso de los huicholes. México: Universidad de Guadalajara.

Jáuregui, Jesús. (1998). La investigación etnológica sobre los coras y huicholes en los últimos cincuenta años: un comentario crítico. En Antropología e historia del Occidente de México (pp. 287-361). México: Sociedad Mexicana de Antropología.

Kindl, Olivia. (2003). La jicara huichola: un microcosmos mesoamericano. México: INAH-Universidad de Guadalajara.

Lamas, Marta. (2015). Usos, dificultades y posibilidades de la categoría de género. En El Género y la Construcción cultural de la diferencia sexual (pp. 327366). México: UNAM.

Lemaistre, Denis. (2003). Le Chamane et son chant: relations ethnographiques d'une expérience parmi les Huicholes du Mexique. Paris: L'Harmattan.

Liffman, Paul. (2012). La territorialidad wixarika y el espacio nacional. Reivindicación indígena en el occidente de México. Zamora Michoacán, México: El Colegio de Michoacán.
López-Austin, Alfredo. (1996). Cuerpo humano e ideología. Las concepciones de los antiguos nahuas. México: Instituto de Investigaciones Antropológicas, Universidad Nacional Autónoma de México.

López-Austin, Alfredo. (2015). Sobre el concepto de cosmovisión. En Alejandra Gámez y Alfredo LópezAustin (Coords.), Cosmovisión mesoamericana: reflexiones, polémicas y etnografías (pp. 17-51). México: El Colegio de México.

López-Austin, Alfredo. (2016). El objeto de estudio. La cosmovisión de la tradición mesoamericana. Arqueología Mexicana, (68), 39-55.

Lumholtz, Carl. (1981). El México desconocido. México: INI.

Lumholtz, Carl. (1986). El arte simbólico y decorativo de los huicholes. México: INI.

Manzanares, Sara. (2009). El Sistema de cargos de los Xukurikate: parentesco y poder en una comunidad Wixarika. México: UNAM.

Marcos, Sylvia. (2012). Raíces epistemológicas mesoamericanas: la construcción religiosa del género. En Religión y Genero. Enciclopedia Iberoamericana de las Religiones, Volumen III (pp. 235-270). Madrid: Editorial Trotta.

Medina, Andrés. (1974). ¿Etnología o literatura? El caso de Benítez y sus indios. Anales de Antropología, 11, 109-140.

Mota, José; Rivera, María y Vizcarra, Ivonne. (2017). El ciclo agrícola y ritual huichol en sinergia con la migración. En Humberto Thomé y William Gómez (Comps.), Estudios de caso sobre ciencias agropecuarias y rurales en el siglo XIX (pp. 148158). Toluca: Editorial UAEMex.

Neurath, Johannes. (2002). Las fiestas de la Casa Grande. Procesos rituales, cosmovisión y estructura social en una comunidad huichola. México: INAH.

Papousek, Dick. (1982). Alfareros-campesinos mazahuas: Situación de estímulo y procesos de adaptación. México: Gobierno del Estado de México.

Pitarch, Pedro. (1996). Ch'ulel: una etnografia de las almas Tzeltales. México: FCE.

Porras, Eugeni. (2003). Consideraciones sobre el neochamanismo y chamanismo huichol. Gaceta de Antropología, 19. Recuperado de: http://www.ugr. es/ pwlac/G19 07Eugeni Porras_Carrillo.html.

Jáuregui, Jesús y Neurath, Johannes (Comps.). (1998). Fiesta, literatura y magia en el Nayarit: ensayos sobre coras, huicholes y mexicaneros de Konrad Theodor Preuss. México: Centro de estudios mexicanos y centroamericanos, Instituto nacional indigenista.

Sánchez-Espíndola, Mónica; Chávez-Mejía, Cristina y Vizcarra-Bordi, Ivonne. (2010). Ahí te encargo el cargo: Empoderamiento o feminización de los sistemas de cargo en la organización de la festividad 
de la virgen de Guadalupe en La Laja, Querétaro. La ventana. Revista de estudios de género, 4(31), 172213.

Sandoval, Fernando. (1994). Familia indigena y unidad doméstica. Los Otomíes del Estado de México. México: UAEM.

Turner, Víctor. (2007). La Selva de los símbolos: aspectos del ritual ndembu. México: Siglo XXI Editores.

Téllez, Víctor. (2005). Xatsitsarie: territorio, gobierno local y ritual en una comunidad huichola. México: El Colegio de Michoacán.

Valdés, Ximena. (2000). Masculinidad en el mundo rural: realidades que cambian, símbolos que permanecen. En José Olavarría y Rodrigo Parrini (Eds.), Masculinidades, Identidad, sexualidad y familia (pp. 29-46). Santiago de Chile: Flacso.
Villa-Rojas, Alfonso. (1961). Notas sobre los huicholes. México: INAH.

Weigand, Phil. (1992). Ensayos sobre el Gran Nayar: entre coras, huicholes y tepehuanos. México: Instituto Nacional Indigenista (INI), Centro de Estudios Mexicanos y Centroamericanos (CEMCA) y El Colegio de Michoacán (COLMICH).

Zingg, Robert. (1998). La mitología de los huicholes. México: El Colegio de Michoacán y El Colegio de Jalisco.

\section{Fuentes primarias}

Entrevista con Ángela, 17 de marzo del 2015.

Entrevista con Enrique L., 15 de junio de 2016. 